\title{
Pulmonary Hypertension and Macrocephaly in a Newborn
}

\author{
Monique Marchand ${ }^{\mathrm{a}} \quad$ Jean-Pierre Pfammatter ${ }^{\mathrm{b}} \quad$ Nicolas Regamey $^{\mathrm{a}}$ \\ Divisions of a Paediatric Respiratory Medicine and ${ }^{b}$ Paediatric Cardiology, Department of Paediatrics, \\ Inselspital and University of Bern, Bern, Switzerland
}

\section{Clinical Presentation - Case Report}

A term-born boy was transferred from a peripheral hospital to our Neonatology Unit on his third day of life for investigation of respiratory distress and need for supplemental oxygen. Pregnancy and birth had been uneventful. Besides tachypnea (respiratory rate 110/min) and macrocephaly (head circumference +2.02 SDS), clinical examination was normal. Echocardiography revealed severe pulmonary hypertension. Underlying structural cardiac disease, significant lung pathology, and neonatal infection were excluded. BMPR-II (bone morphogenic protein receptor type II) gene analysis to rule out familial pulmonary hypertension was normal. After glutaraciduria had been excluded, macrocephaly was interpreted as familial, as it was present in both parents. The boy was discharged home on his third week of life with a presumptive diagnosis of primary pulmonary hypertension of the newborn (PPHN). Tachypnea was persistent but the respiratory rate had decreased to 55/ min. Supplemental oxygen (0.3-0.5 liters $/ \mathrm{min})$ was still necessary to maintain normal blood oxygen saturation, and a nasogastric tube was put in place because of poor feeding and weight gain. Home cardiopulmonary monitoring was installed.

\section{KARGER}

Fax +4161306 1234

E-Mail karger@karger.ch

www.karger.com
(C) 2012 S. Karger AG, Basel

0025-7931/12/0835-0421\$38.00/0

Accessible online at:

www.karger.com/res

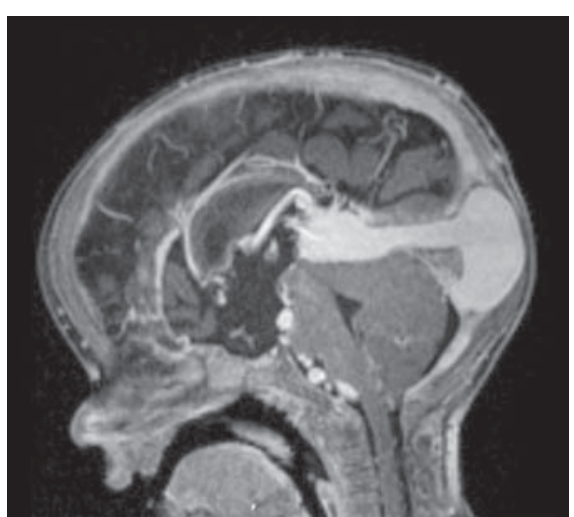

Fig. 1. Magnetic resonance angiography of the neurocranium.

Three weeks later, the boy was still tachypneic and needed supplemental oxygen and nasogastric tube feeding. Cardiac catheterization was performed and showed normal pulmonary arterial pressure but left ventricle dilatation and high-output (8.4 liters/min) cardiac failure. Distended veins coming from the brain were seen and subsequently magnetic resonance angiography of the neurocranium was performed (fig. 1).

What is your diagnosis? 
Figure 1 shows a vein of Galen aneurismal malformation (VGAM). In our case, this malformation led to congestive high-output heart failure and pulmonary hypertension. The aneurismal malformation was embolized at the ages of 6 and 7 months. Follow-up at 30 months showed normal neurologic development but persistent hydrocephalus.

PPHN occurs in about 2 per 1,000 live births [1]. Its etiology often remains unclear, but common causes include structural lung disease (e.g. congenital diaphragmatic hernia), lung pathology (e.g. meconium aspiration pneumonia, hyaline membrane disease), and group B streptococcal infection. VGAM are congenital cerebral vascular malformations characterized by multiple arteriovenous connections draining into an enlarged median vein. They represent only $1 \%$ of all intracranial vascular malformations but $30 \%$ of vascular malformations presenting in the pediatric age group [2]. VGAM typically manifest as congestive high-output heart failure. During intrauterine life, blood flow through a VGAM is low because of the low resistance of the placental circulation. After birth, blood pressures rise and flow through the VGAM increases, leading to a compensatory increase in cardiac output to maintain adequate perfusion of the systemic vasculature. Pulmonary hypertension, which results from the excessive blood flow across pulmonary vessels, can be a presenting sign of VGAM [3]. Diagnosis is made either by ante- or postnatal ultrasound or by magnetic resonance angiography of the brain after birth. Upon diagnosis, rapid management of the cardiac failure is essential. Closure of the VGAM by arterial or venous embolization (by coil or glue) is usually done at the age of 5-6 months. Outcomes vary from good outcomes without neurological deficits to death.

Diagnosis in our case could have been made clinically if the skull had been auscultated with a stethoscope. The systolo-diastolic flow murmur caused by the arteriovenous connections was audible over the infant's whole skull. The combination of PPHN with macrocephaly should ring a bell in every physician's head and prompt for the search for arteriovenous cerebral malformations.

\section{Key Words}

Pulmonary hypertension - Macrocephaly · Newborn • Venous malformation • Vena galeni 